\title{
Psychokardiologie: Wie Herz und Psyche zusammenhängen
}

\author{
Maxie Bunz, Ingrid Kindermann, Julia Karbach, Sonja Wedegärtner, Michael Böhm, Denise Lenski
}

Patienten mit Herzerkrankungen leiden häufig unter psychischen Komorbiditäten. Diese senken die Lebensqualität der Betroffenen und wirken sich negativ auf Entstehung und Verlauf von Herzerkrankungen aus. Insbesondere Depression, Angst und posttraumatische Belastungsstörung (PTBS) können die Hospitalisierungs-, Morbiditäts- und Mortalitätsrate ansteigen lassen. Eine frühe Diagnose und Behandlung der psychischen Störungen sind unumgänglich. Die Psychokardiologie befasst sich mit dem Zusammenspiel kardialer Erkrankungen und psychischer Veränderungen sowie mit

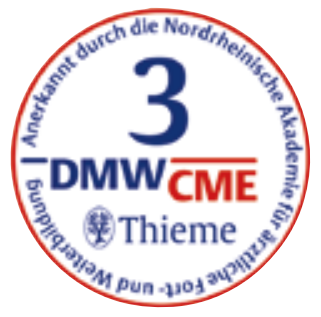
den darauf einwirkenden psychosozialen Faktoren.

\section{Der konkrete Fall}

Herr M., ein 55-jähriger Patient, kommt nach mehrfachen adäquaten Schockabgaben seines implantierten Cardioverter Defibrillators (ICD) in psychotherapeutische Behandlung.

Anamnese | Bei dem Patienten wurde 9 Jahre zuvor primärprophylaktisch ein ICD implantiert. Grund war eine ischämische Kardiomyopathie mit hochgradig eingeschränkter linksventrikulärer Pumpfunktion nach Hinterwandinfarkt. Er berichtet über einen Kreislaufkollaps nach multiplen adäquaten Schockabgaben infolge ventrikulärer Tachykardien - einem sog. elektrischen Sturm. Der Zusammenbruch ereignete sich am Straßenrand. Trotz mehrfacher Hilferufe kam erst nach 15 min zufällig eine Ärztin vorbei, die ihn notfallmäßig versorgte. Herr M. empfand die Schockabgaben als extrem schmerzhaft. Er hatte Angst - sogar Todesangst Schlafstörungen und Alpträume nach diesem Ereignis. Seither halte er sich fast ausschließlich im Haus auf, um ähnliche Situationen und Erinnerungen zu vermeiden. Auch immer wiederkehrende Erinnerungen an das Erlebnis quälten ihn.

Diagnose und Behandlung I Der Patient leidet an einer posttraumatischen Belastungsstörung (PTBS). Behandlungsschwerpunkte liegen zunächst auf dem Abbau von Ängsten bei körperlicher Belastung und auf einer Steigerung der Anpassungsfähigkeit an das Gerät.

Konfrontationstherapie I Nach Stabilisierung des Patienten beginnt eine Traumatherapie mittels kognitiver, konfrontativer und imaginativer Techniken: Wesentlicher Inhalt der Therapie ist die Konfrontation mit dem traumatischen Ereignis, zunächst nur in der Vorstellung des Patienten (,in sensu“). Dann sucht er mit Unterstützung der Therapeutin den Ort des Geschehens auf - für den Patienten ein hoch belastendes Erlebnis. Er befand sich dabei in einem kör- perlich und emotional stark erregten Zustand. Ziel dieser Konfrontationstherapie ist, dass Herr M. die Exposition und die Übererregung („Hyperarousal“) aushält, um damit die Anspannung zu reduzieren. Diesen Schritt meistert er erfolgreich.

Rescripting-Therapie | Danach gelingt ihm auch das sog. „Rescripting“: Als eigener Regisseur schreibt er das traumatische Ereignis so um, dass er ihm damit die Bedrohlichkeit nimmt. Nach Konfrontations- und Rescripting-Therapie geht die PTBS-Symptomatik deutlich zurück. Herr M. leidet seitdem nicht mehr unter Alpträumen, ist ruhiger geworden und traut sich wieder mehr zu. Auch die ständigen Bilder des Ereignisses sind nur noch selten präsent. Wenn sie doch auftreten, weiß er, wie er damit umgehen kann.

\section{Herzerkrankung und Psyche}

Psychokardiologie | Kardiovaskuläre Erkrankungen sind die häufigste Todesursache in der westlichen Welt [1]. Herzerkrankungen wie die

- koronare Herzkrankheit,

- Herzinsuffizienz oder

- Rhythmusstörungen

können je nach Schweregrad der Erkrankung die kognitive Funktionalität, das psychische Wohlbefinden und die Lebensqualität der Betroffenen erheblich beeinträchtigen. Die Psychokardiologie beschäftigt sich mit dem Zusammenhang von Herzkrankheiten und psychischen Störungen, da diese sich reziprok beeinflussen können.

Risikofaktoren | Risikofaktoren für die Entstehung kardiovaskulärer Erkrankungen sind

- arterielle Hypertonie,

- Hyperlipidämie,

- Rauchen,

- Diabetes mellitus und

- Adipositas. 
Dabei sind psychische Störungen wie z. B. Depression und Angststörungen eigenständige kardiale Risikofaktoren. Psychosoziale Belastungen und chronischer Stress können zudem auch die klassischen Risiken verstärken: Eine betroffene Person raucht vielleicht vermehrt, isst mehr und nimmt dementsprechend zu oder hat hypertensive Blutdruckwerte. Solche Belastungen tragen somit zur Entwicklung von Herzerkrankungen bei.

Der Einfluss psychischer Faktoren auf die Entstehung und den Verlauf kardialer Erkrankungen wird oftmals übersehen.

Psychische Störungen I Die psychische Gesundheit ist eng mit der Funktionalität des Herzens verbunden. Psychische Beschwerden können zu unterschiedlichen Zeitpunkten im Krankheitsverlauf auftreten: Sie können

- als eigenständiger Risikofaktor zur Entstehung der Krankheit beitragen,

- als komorbide Erkrankung den Krankheitsverlauf negativ beeinflussen oder

- durch die kardiale Erkrankung ausgelöst oder verstärkt werden.

Eine psychische Störung kann die Lebensqualität der Betroffenen erheblich beeinträchtigen. Besonders

- Depression,

- Angststörungen,

- PTBS,

- Stress und

- kognitive Einschränkungen bis hin zur Demenz beeinflussen den Verlauf von Herzerkrankungen. Dennoch werden psychologische Aspekte von Herzerkrankungen oft vernachlässigt.

\section{Koronare Herzkrankheit (KHK)}

Risikofaktoren | Relevante psychosoziale Risikoindikatoren der koronaren Herzerkrankung (KHK) sind ein niedriger sozioökonomischer Status und chronischer Stress am Arbeitsplatz wie z.B.

- eine niedrige berufliche Hierarchieposition,

- hohe Arbeitsanforderungen bei niedrigem Handlungsspielraum,

- dauerhafte Schichtarbeit und

- die Doppelbelastung durch Familie und Beruf [2]. Auch geringe soziale Unterstützung vergrößert die Wahrscheinlichkeit, dass eine KHK auftritt und ist bei vorbestehender KHK mit einer erhöhten Mortalität assoziiert [3]. Die Einstellung des Partners gegenüber der Erkrankung und dessen Verhalten beeinflussen in hohem Maß das Gesundheits- und Krankheitsverhalten der Betroffenen. Patienten mit überfürsorglichen Lebenspartnern etwa berichten eine geringere Lebensqualität und höhere psychische Belastung [4].

Angst | Psychische Störungen wie Angststörungen und Depression üben einen negativen Einfluss auf die Entwicklung und den Verlauf von kardiologischen Erkrankungen aus. Sie können aber auch selbst durch kardiologische Erkrankungen hervorgerufen oder in ihrer Entstehung begünstigt werden. Angst erhöht laut einer Metaanalyse von Roest et al. das Risiko für eine KHK um 26\% und das Mortalitätsrisiko um 48\% [5]. Akute psychische Belastungen können sich aber auch zusätzlich negativ auf das Krankheitsverhalten auswirken, indem die Patienten Symptome

- vermindert wahrnehmen,

- fehlinterpretieren oder

- verleugnen.

So kann Angst z.B. die Schmerzwahrnehmung senken [6], wodurch die Betroffenen verspätet ärztliche Hilfe aufsuchen.

PTBS | Insbesondere nach akutem Myokardinfarkt können Patienten eine PTBS entwickeln [7]. Sie erleben häufig „Flashbacks“ - sich aufdrängende, wiederholte Erinnerungen an das traumatisierende Ereignis - und vermeiden Situationen, die sie mit dem Herzinfarkt in Verbindung bringen. Oft leiden die Betroffenen auch an vegetativer Übererregtheit mit übersteigerter Schreckhaftigkeit und Schlafstörungen. Die PTBS nach kardialem Ereignis ist mit einer deutlich verringerten Lebensqualität assoziiert - die Wahrscheinlichkeit einer kardiovaskulären Rehospitalisierung innerhalb eines Jahres ist mehr als doppelt so hoch [7].

Depression und KHK | Depressive Symptome mit Gefühlen der Niedergeschlagenheit, Hoffnungslosigkeit und Antriebslosigkeit treten bei $20-50 \%$ der Patienten mit KHK auf. Bei 15-20\% der Betroffenen liegt eine ausgeprägte klinische Symptomatik im Sinne einer Depression vor [2]. Diese ist geprägt von einer anhaltenden depressiven Verstimmung über mehr als 2 Wochen mit Interessen- und Freudlosigkeit. Das Risiko für eine Depression ist bei Frauen unter 60 Jahren nach einem Herzinfarkt mit bis zu $40 \%$ besonders hoch [8]. Depressive Symptome steigern wiederum das Risiko für eine koronare Herzerkrankung und haben einen negativen Effekt auf den Krankheitsverlauf [2].

Der bidirektionale Zusammenhang zwischen KHK und Depression ist besonders problematisch, da Depression und Depressivität zum einen das Risiko für eine koronare Herzerkrankung steigern. Zum anderen können sie aber auch aus einer Herzerkrankung resultieren und die Prognose beeinträchtigen.

Sowohl biologische als auch Verhaltensprozesse erklären die Verbindung von Depression und kardialen Ereignissen. Einerseits können pathophysiologische Veränderungen bei Patienten mit Depression zu einer Progression der Arteriosklerose führen [9]. Von zentraler Bedeutung sind hierbei: 
- der Einfluss einer chronisch erhöhten Aktivität des sympathischen Nervensystems

- Dysfunktionen des endokrinen Systems wie eine gesteigerte Aktivierung der Hypothalamus-Hypophysen-Nebennierenrinden-Achse

Des Weiteren ist Depression mit inflammatorischen Prozessen assoziiert, die zu einem ungünstigen Verlauf beitragen: z. B. erhöhte Konzentrationen von Interleukin-6 und C-reaktivem Protein und eine vermehrte Thrombozytenaktivität. Andererseits werden

- Ernährung,

- Tabak- und Alkoholkonsum,

- Bewegungsmangel,

- soziale Isolation und

- verringerte Medikamentenadhärenz

als Mediatorvariablen diskutiert [2, 10, 9].

\section{Herzrhythmusstörungen}

Panikattacken oder Rhythmusstörungen? | Auch bei Herzrhythmusstörungen besteht ein bidirektionaler Zusammenhang zwischen psychischen und körperlichen Symptomen: Psychische Störungen wie Angst und Panikstörung können Herzrhythmusstörungen auslösen, aber auch durch sie verursacht werden. Zu den häufigen Rhythmusstörungen, die mit psychischen Störungen assoziiert sind, zählen

- Extrasystolen,

- supraventrikuläre Tachykardien (SVT),

- Vorhofflimmern und Vorhofflattern sowie

- ventrikuläre Rhythmusstörungen wie Kammerflimmern und Kammerflattern.
Extrasystolen und SVT - wie z. B. AV-nodale ReentryTachykardien - führen wegen der Ähnlichkeit mit Symptomen einer Panikstörung häufig zu einer Fehldiagnose. Sie können aufgrund einer spontanen Terminierung als Panikattacken fehlgedeutet werden: Zum einen sind durch die kurze Dauer im anschließenden EKG keine Veränderungen mehr sichtbar und zum anderen spüren Patienten in dieser Situation oft starke Angst. Umgekehrt kann man Symptome einer Panikattacke, wie starkes Herzklopfen oder -rasen, als SVT missinterpretieren. Zur Aufklärung sollte man daher eine genaue Anamnese der Krankheitsgeschichte und Symptome vornehmen und ein Langzeit-EKG aufzeichnen [11].

Teufelskreis der Angst | Bei Panikstörungen nehmen die Patienten während eines Panikanfalls körperliche Veränderungen wahr und bewerten sie als gefährlich. Diese Bewertung erzeugt bei ihnen starke Angst, die zu physiologischen Veränderungen wie z.B. erhöhter Atemfrequenz und steigendem Blutdruck führt - wiederum Ereignisse, die der Patient als bedrohlich bewertet. Es entsteht ein Circulus vitiosus der Angst ( Abb. 1). Bei Patienten mit einem schwerwiegenden kardialen Ereignis in der Anamnese, wie z. B. einem Herzinfarkt, kann die verschärfte Wahrnehmung von Vorgängen im Körperinneren (Interozeption) einen solchen Circulus vitiosus auslösen. Dieser Kreislauf kann dazu führen, dass die Betroffenen ihre Arbeit nicht mehr ausüben können und körperliche Aktivität gänzlich meiden.

Implantierbarer Cardioverter Defibrillator (ICD) | Eine evidenzbasierte Behandlung lebensgefährlicher Herzrhythmusstörungen ist neben der me-

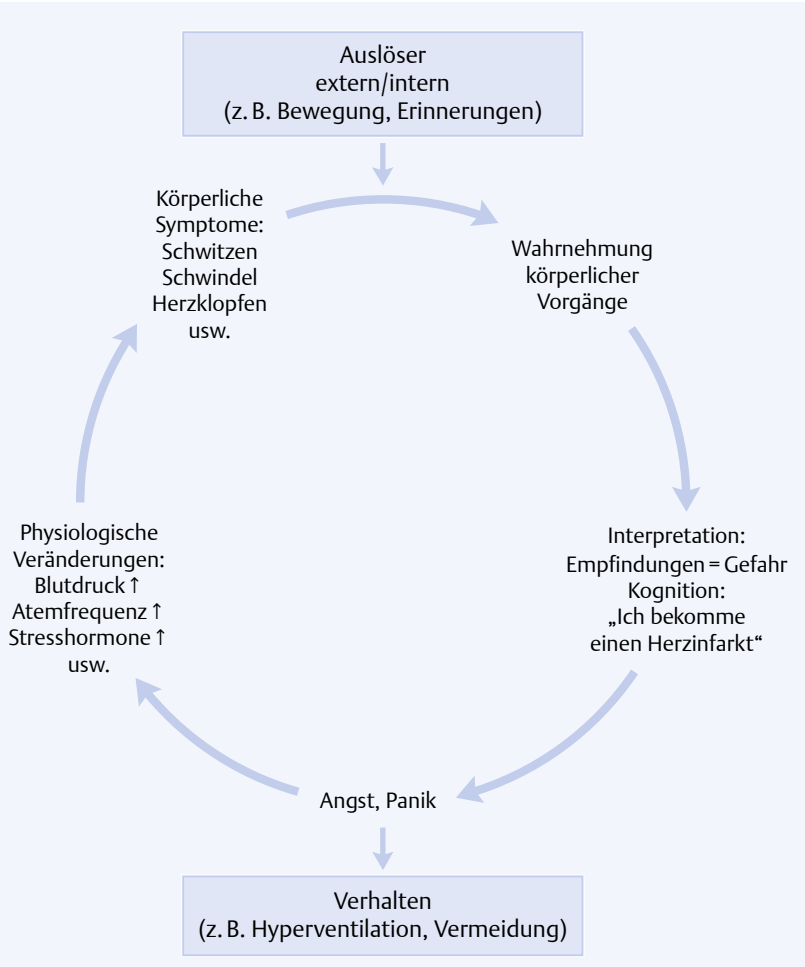

Abb. 1 Circulus vitiosus der Angst. Bildnachweis: Maxie Bunz, modifiziert nach Margraf \& Schneider 2009 [12] 
dikamentösen Therapie die Implantation eines Cardioverter Defibrillators (ICD). Nach Implantation des ICD sind die Patienten häufig erst einmal erleichtert. Das Einsetzen des ICD erfordert jedoch von den Erkrankten auch eine enorme Anpassungsleistung:

- Auseinandersetzung mit der Abhängigkeit von dem Gerät

- Fremdkörpergefühle

- Veränderungen des beruflichen und familiären Umfelds

Die Prävalenzraten für klinisch bedeutsame Depressionen und Angststörungen nach ICD-Implantation von jeweils etwa $20 \%$ sind vergleichbar mit der Prävalenz nach Myokardinfarkt [13]. Die Bedeutung des ICD für die Entstehung psychischer Störungen wird jedoch kontrovers diskutiert und bedarf weiterer wissenschaftlicher Untersuchungen.

Die psychische Belastung der Patienten kann besonders bei Schockabgaben oder Komplikationen aufgrund der ICD-Implantation wie

- Tascheneinblutungen,

- Sondendefekte und

- inadäquate ICD-Entladungen deutlich ansteigen.

Frühe Therapie | Psychische Komorbiditäten sind bei ICD-Trägern nicht zu unterschätzen: Sie wirken sich nicht nur negativ auf die Lebensqualität aus, sondern Depression und PTBS erhöhen auch das Risiko für die Mortalität der Patienten [14, 15]. Eine persistierende PTBS tritt bei fast $20 \%$ der Patienten nach Implantation auf [16]. Deshalb ist es Aufgabe des behandelnden Arztes, den Patienten hinsichtlich einer potenziellen Traumatisierung, - insbesondere infolge einer Schockabgabe des Geräts - zu befragen. Bei Verdacht auf PTBS sollte ein weiterführendes Screening und die fachärztliche Abklärung erfolgen, um ggf. eine frühe psychotherapeutische Behandlung einzuleiten.

\section{Herzinsuffizienz}

Depression I Patienten mit Herzinsuffizienz leiden häufig unter ängstlich-depressiven Symptomen. Ergebnisse aus Metaanalysen zeigen: Die Prävalenz einer Depression in dieser Patientengruppe liegt bei $21,5 \%$ [17]. Je ausgeprägter die Herzinsuffizienz (Schweregrad nach NYHA-Klasse, New York Heart Association), desto höher ist der Anteil von Erkrankten mit depressiven Störungen [17]. Dabei

- erhöhen depressive Symptome die Hospitalisierungsrate,

- verdoppeln das Risiko für nachfolgende klinische Ereignisse und Mortalität [17] und

- führen zu einer deutlichen Beeinträchtigung der Lebensqualität [18].
Aufgrund der hohen Anzahl Betroffener und der schwerwiegenden Implikationen sollten Sie eine depressive Störung immer abklären. Sie wird im klinischen Alltag oft übersehen, weil die betroffenen Patienten sich zurückziehen und weniger offensichtlich ihre Emotionen äußern. Häufig zeigen sie zudem eine ausgeprägte Leugnungsneigung oder schämen sich wegen der erlebten eigenen Schwäche. Die differenzialdiagnostische Abklärung zu Symptomen der Herzinsuffizienz wie z.B. Müdigkeit, Erschöpfung, Appetitmangel und Schlafstörungen stellt darüber hinaus hohe Anforderungen an die behandelnden Hausärzte und Kardiologen.

Kognition | Herzinsuffiziente zeigen im Vergleich zu einer gesunden Probandengruppe ein 1,6-fach höheres Risiko für kognitive Beeinträchtigungen [19]. Depressionen scheinen diese verminderte kognitive Leistungsfähigkeit noch zu verstärken [20]. Kognitive Dysfunktionen äußern sich bei Herzinsuffizienten u.a. in Form von

- Gedächtnisdefiziten,

- Störungen exekutiver Funktionen (Fähigkeiten wie Planen, Problemlösen, Initiation und Inhibition von Handlungen) und

- verlangsamter Verarbeitungsgeschwindigkeit.

Dekompensierte Patienten weisen die stärksten Beeinträchtigungen auf [21]. Entscheidungsschwierigkeiten und verlangsamtes Handeln verstärken die Auswirkungen einer generell verminderten Adhärenz bei Herzinsuffizienten und führen zu einem verschlechterten Krankheitsmanagement: Nur 40-80\% der Patienten nehmen regelmäßig ihre Medikamente [22]. In der Folge kommt es zu vermehrten Komplikationen, wie z.B. Dekompensationen und Rehospitalisierungen.

Ursachen kognitiver Defizite | Die Ätiopathogenese der kognitiven Defizite ist multifaktoriell. So werden u.a.

- eine verminderte zerebrale Durchblutung,

- rezidivierende Hypotonien,

- zerebrale Infarkte,

- hormonelle Ursachen (Hypothyreose) und

- psychosoziale Faktoren wie der sozioökonomische Status

als Ursachen diskutiert [23].

Wenn beim Patienten Verständnis- und Adhärenzprobleme auftreten, sollten Sie mögliche kognitive Dysfunktionen differenzialdiagnostisch in Betracht ziehen und weiter abklären.

\section{Diagnostik und Intervention bei psychischen Störungen}

Vorgehen | Die Diagnostik psychischer Störungen bei kardiologischen Patienten ist entscheidend, um betroffene Personen zu identifizieren und 
eine adäquate Behandlung einleiten zu können. Die Ergebnisse des Screenings geben jedoch lediglich einen Hinweis auf eine möglicherweise vorliegende psychische Störung - sie ersetzen nicht die klinische Diagnose. Diese sollten nur ausgebildete Psychotherapeuten oder Fachärzte stellen. Um erste Hinweise auf eine psychische Störung zu erhalten, sollten die behandelnden Ärzte im Gespräch mit dem Patienten

- chronische Stressoren,

- emotionale Faktoren und

- somatische Beschwerden wie z.B. Erschöpfung und Schlafstörungen

thematisieren [9]. Zur Abklärung einer möglichen Traumatisierung eignet sich diese Frage: „Hatten Sie in letzter Zeit verstärkt Erinnerungen an eine Situation, in der Sie sich sehr bedroht fühlten?"

Die Nationale Versorgungsleitlinie Unipolare Depression [24] empfiehlt den Zwei-Fragen-Test [25] zur initialen Abklärung einer möglichen Depression. Wenn ein Patient die folgenden 2 Fragen mit „Ja“ beantwortet, sollten Sie eine ausführliche Depressionsdiagnostik veranlassen:

- „Fühlten Sie sich im letzten Monat häufig niedergeschlagen, traurig, bedrückt oder hoffnungslos?"

- „Hatten Sie im letzten Monat deutlich weniger Lust und Freude an Dingen, die Sie sonst gerne tun?"

Screeninginstrumente | Bei Verdacht auf psychische Störungen sollte ein Screening mit Hilfe von Fragebögen erfolgen. Bestätigen die Fragebögen den Verdacht, sollte die Diagnose auf Basis eines strukturierten klinischen Interviews gestellt werden. Dieses können Psychotherapeuten, Psychiater oder Psychosomatiker mit dem Patienten führen. Als Screeninginstrument zur simultanen Erfassung von Depressivität und Angst bietet sich die deutsche Version des Fragebogens „Hospital Anxiety and Depression Scale“ (HADS-D) an [26]. Die „Impact of Event Skala - revidierte Version“ (IES-R) eignet sich zur Erfassung einer möglichen PTBS [27]. Zur Abklärung einer möglichen Demenz wird der Test „DemTect“ empfohlen [28].

Stigmatisierung | Es ist wichtig, eine Stigmatisierung des Patienten als „psychisch krank“ zu vermeiden. Man sollte dem Patienten vermitteln, dass psychische Beschwerden gehäuft auftreten, durch die kardiale Erkrankung verursacht sein können und meist gut zu behandeln sind.

Pharmakologie | Bei der pharmakologischen Therapie von psychischen Störungen sind regelmäßige EKG- und Laborkontrollen unabdingbar, da z.B. antidepressiv wirkende selektive Serotonin-Wiederaufnahmehemmer (SSRI) das QTIntervall verlängern und somit das Risiko für Herzrhythmusstörungen erhöhen können. Trizy- klische Antidepressiva sollten aufgrund arrhythmogener Effekte vermieden werden.

Berücksichtigen Sie bei einer psychopharmakologischen antidepressiven Therapie immer die kardiale Grunderkrankung, Wechselwirkungen mit anderen Medikamenten und unerwünschte Nebenwirkungen.

Auswirkung von Psychotherapie | Der Nutzen einer psychotherapeutischen Intervention auf den Verlauf der kardiologischen Erkrankung ist noch nicht ausreichend erforscht. Jedoch gibt es Hinweise darauf, dass die kardiale Mortalität abnimmt, wenn die Psychotherapie die psychische Belastung verringert [9]. Positive Effekte auf Depression, Angst und PTBS wurden - bei hoher Kosten-Effektivität - nachgewiesen [29, 30].

Liegen Depression, Angst oder PTBS vor, ist eine psychotherapeutische Behandlung bei Menschen mit Herzerkrankung empfehlenswert.

Interventionen | Im Anschluss an die Diagnostik steht die Auswahl der geeigneten Interventionen. Zur Förderung der Adhärenz haben sich

- Schulungen zur Krankheitsbewältigung,

- individuelle Medikamenten-Dosierhilfen,

- regelmäßige Kontrolluntersuchungen und

- die Einbindung von Angehörigen

bewährt. Die psychische Belastung und das entsprechende Verhalten von Angehörigen kann das Befinden der Patienten nachhaltig beeinflussen. Daher sollte man die Aufklärung und psychologische Betreuung der Angehörigen mit in den Behandlungsprozess einbeziehen. Im Zuge einer Rehabilitations-Maßnahme haben sich 2 Konzepte als besonders hilfreich erwiesen: das Konzept der motivierenden Gesprächsführung zur Förderung der intrinsischen Motivation zur Verhaltensänderung [31] und das Konzept der Handlungs- und Bewältigungsplanung zur Entwicklung von Selbstregulationsstrategien [32]. Diese Strategien sollen dem Patienten bei der Umsetzung von gesundheitsförderlichem Verhalten helfen [33]. Außerdem können

- Stressmanagementtechniken,

- Entspannungsverfahren wie autogenes Training und progressive Muskelentspannung sowie

- körperliche Aktivität

zur Entlastung der Patienten beitragen.

Multimodale Behandlung I Klinisch relevante psychische Störungen sollten grundsätzlich psychotherapeutisch und/oder medikamentös behandelt werden. Die Aufklärung der kardiologischen Ärzte und Pflegekräfte hinsichtlich psychosozialer Belastungsfaktoren, psychischer Störungen und sinnvoller Behandlungsmaßnahmen ist notwendig, um eine multimodale Therapie zu ermöglichen. 


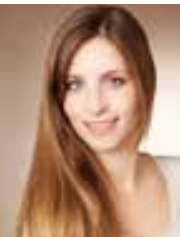

Dipl.-Psych. Maxie Bunz ist Psychologin an der Klinik für Innere Medizin III des Universitätsklinikum des Saarlandes Kindermann ist Oberärztin an der Klinik für Innere Medizin III des Universitätsklinikum des Saarlandes

Prof. Dr. Julia Karbach ist Leiterin der Pädagogischen Psychologie an der Universität des Saarlandes, Fachrichtung Bildungswissenschaften

Dipl.-Psych. Sonja Wedegärtner ist Psychologin an der Klinik für Innere Medizin III des Universitätsklinikum des Saarlandes

Prof. Dr. med. Michael Böhm ist Direktor der Klinik für Innere Medizin III des Universitätsklini-

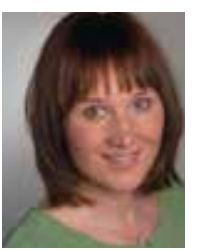

Dr. rer. med. Dipl.-Psych. Denise Lenski

ist Psychologin an der Klinik für Innere Medizin III des Universitätsklinikum des Saarlandes
PD Dr. med. Ingrid kum des Saarlandes

Auswirkungen psychischer Störungen bei

Patienten mit Herzerkrankungen

- geringere Lebensqualität

- Überdiagnostik und hohe ökonomische Belastung

- verminderte Adhärenz

- schlechteres Krankheitsmanagement

- erhöhtes Risiko für erneute kardiale Ereignisse

- erhöhtes Mortalitätsrisiko

\section{Sonderfall Stresskardiomyopathie (Tako-Tsubo-Syndrom)}

Die Tako-Tsubo-Kardiomyopathie - auch Stresskardiomyopathie, Broken-Heart-Syndrom und transiente linksventrikuläre apikale Ballonierung genannt - ist eine Sonderform der Kardiomyopathie. Sie tritt meist nach einem emotional hoch belastenden Lebensereignis wie nach Verlust des Lebenspartners oder nach starken familiären und beruflichen Belastungen auf. Die Prävalenz liegt bei $1-2 \%$ aller Patienten mit akutem Koronarsyndrom [34]. Klinisch ähnelt das Krankheitsbild einem akuten Myokardinfarkt mit plötzlich auftretender

- Angina pectoris,

- EKG-Veränderungen (ST-Strecken-Hebungen oder T-Wellen-Inversion) und

- Ausschüttung kardialer Nekrosemarker (Kreatinkinase, Troponin T) [35].

Pathognomonisch für diese Erkrankung ist eine vorübergehende apikale Hypokinesie (sog. apikales Ballooning) des linken Ventrikels bei basaler Hyperkontraktilität. Dabei erinnert die Form des linken Ventrikels an den Tako-Tsubo - eine japanische Oktopusfalle. Koronarangiografisch zeigt sich im Gegensatz zum akuten Infarkt ein freies Kranzgefäßsystem. Die zugrundeliegenden pathophysiologischen Mechanismen des TakoTsubo-Syndroms sind nicht eindeutig bekannt. Als Ursache diskutiert man u.a. Koronarspasmen durch Ausschüttung von Stresshormonen wie Adrenalin und Noradrenalin [36]. Die meisten Patienten erholen sich innerhalb weniger Tage mit einer Restitutio ad integrum. Bei der Behandlung der Stresskardiomyopathie sollten Sie mit einem Psychologen oder Psychotherapeuten kooperieren - auch um Rezidiven vorzubeugen.

\section{Fazit}

Interessenkonflikt

Die Autoren geben an, dass

kein Interessenkonflikt besteht.

DOI 10.1055/s-0040-100440 VNR 2760512015147121756 Dtsch Med Wochenschr 2015; 140: 117-124

(C) Georg Thieme Verlag KG . Stuttgart · New York ·

ISSN 0012-0472

leiden häufig unter psychischen Beschwerden und kognitiven Dysfunktionen. Für die Prognose ist die simultane Behandlung der kardiologischen und der psychischen Erkrankung besonders wichtig - sie vergrößert die Chance auf Rehabilitation erheblich. Ein Screening auf psychische Komorbiditäten sollte bei kardiologischen Patienten deshalb standardmäßig erfolgen. Die Psychokardio- logie verfolgt dabei einen ganzheitlichen Ansatz, um dem Zusammenspiel von somatischem und psychischem Status der Patienten gerecht zu werden.

\section{Konsequenz für Klinik und Praxis}

- Bei Patienten mit kardiovaskulären Erkrankungen treten besonders häufig Angst, Panikstörung, posttraumatische Belastungsstörung (PTBS) und Depression auf.

- Die Behandlung dieser psychischen Komorbiditäten beeinflusst den weiteren Krankheitsverlauf.

- Behandelnde Ärzte sollten daher auf psychische Störungen achten.

- Bei Verdacht sollte ein Fragebogen-Screening und ggf. die Einleitung einer weiterführenden Diagnostik und Therapie durch Psychotherapeuten und Psychiater erfolgen.

- Die Aufklärung kardiologischen Fachpersonals über psychologische Faktoren ist essenziell

- für eine frühzeitige Erkennung,

> für eine multimodale Therapie und zur Vermeidung einer Stigmatisierung.

\section{Literatur}

1 Lozano R, Naghavi M, Foreman K et al. Global and regional mortality from 235 causes of death for 20 age groups in 1990 and 2010: a systematic analysis for the Global Burden of Disease Study 2010. Lancet 2013; 380: 2095-2128

2 Ladwig K-H, Lederbogen F, Albus C et al. Positionspapier zur Bedeutung psychosozialer Faktoren in der Kardiologie. Der Kardiologe 2013; 7: 7-27

3 Barth J, Schneider S, von Känel R. Lack of social support in the etiology and the prognosis of coronary heart disease: a systematic review and meta-analysis. Psychosom Med 2010; 72: 229-238

4 Joekes K, Van Elderen T, Schreurs K. Self-efficacy and overprotection are related to quality of life, psychological well-being and self-management in cardiac patients. J Health Psychol 2007; 12: 4-16

5 Roest AM, Martens EJ, de Jonge P et al. Anxiety and risk of incident coronary heart disease. A meta-analysis. J Am Coll Cardiol 2010; 56: 38-46

6 Ladwig K-H, Gärtner C, Walz LM et al. Die innere Barriere: der Beitrag gesundheitspsychologischer Konzepte zur Erklärung der prähospitalen Verzögerungszeit beim akuten Myokardinfarkt. Psychother Psych Med 2009; 59: 440-445

7 Shemesh E, Yehuda R, Milo O et al. Posttraumatic stress, nonadherence, and adverse outcome in survivors of a myocardial infarction. Psychosom Med 2004; 66: 521-526

8 Mallik S, Spertus JA, Reid KJ et al. Depressive symptoms after acute myocardial infarction: Evidence for highest rates in younger women. Arch Intern Med 2006; 166 $876-883$

9 Rozanski A, Blumenthal JA, Davidson KW et al. The epidemiology, pathophysiology, and management of psychosocial risk factors in cardiac practice. The emerging field of behavioral cardiology. J Am Coll Cardiol 2005; 45: 637-651
Vollständiges Literaturverzeichnis unter http://dx.doi.org/10.1055/s-0040-100440 
10 Lichtman JH, Bigger JT, Blumenthal JA et al. Depression and coronary heart disease: recommendations for screening, referral, and treatment: a science advisory from the American Heart Association Prevention Committee of the Council on Cardiovascular Nursing, Council on Clinical Cardiology, Council on Epidemiology and Prevention, and Interdisciplinary Council on Quality of Care and Outcomes Research: endorsed by the American Psychiatric Association. Circulation 2008; 118: 1768-1775

11 Frommeyer G, Eckardt L, Breithardt G. Panic attacks and supraventricular tachycardias: the chicken or the egg? Neth Heart J 2013; 21: 74-77

12 Margraf J, Schneider S. Lehrbuch der Verhaltenstherapie. Heidelberg: Springer Verlag; 2009

13 Magyar-Russell G, Thombs BD, Cai JX et al. The prevalence of anxiety and depression in adults with implantable cardioverter defibrillators: a systematic review. J Psychosom Res 2011; 71: 223-231

14 Ladwig K-H, Baumert J, Marten-Mittag B et al. Posttraumatic stress symptoms and predicted mortality in patients with implantable cardioverter-defibrillators: results from the prospective living with an implanted cardioverter-defibrillator study. Arch Gen Psychiatry 2008; 65: 1324-1330

15 Tzeis S, Kolb C, Baumert J et al. Effect of depression on mortality in implantable Cardioverter Defibrillator recipients - Findings from the prospective LICAD study. PACE 2011; 34: 991-997

16 von Känel R, Baumert J, Kolb C et al. Chronic posttraumatic stress and its predictors in patients living with an implantable cardioverter defibrillator. J Affect Disorders 2011; 131: 344-352

17 Rutledge T, Reis VA, Linke SE et al. Depression in heart failure. A meta-analytic review of prevalence, intervention effects, and associations with clinical outcomes. J Am Coll Cardiol 2006; 48: 1527-1537

18 Faller H, Störk S, Schuler M et al. Depression and disease severity as predictors of health-related quality of life in patients with chronic heart failure $-\mathrm{a}$ structural equation modeling approach. J Card Fail 2009; 15: 286-292

19 Vogels RLC, Scheltens P, Schroeder-Tanka JM et al. Cognitive impairment in heart failure: a systematic review of the literature. Eur J Heart Fail 2007; 9: 440-449

20 Alves TC, Rays J, Telles R et al. Effects of antidepressant treatment on cognitive performance in elderly subjects with heart failure and comorbid major depression: an exploratory study. Psychosomatics 2007; 48: 22-30

21 Kindermann I, Fischer D, Karbach J et al. Cognitive function in patients with decompensated heart failure: the Cognitive Impairment in Heart Failure (CoglmpairHF) study. Eur J Heart Fail 2012; 14: 404-413
22 Riles EM, Jain AV, Fendrick AM. Medication adherence and heart failure. Curr Cardiol Rep 2014; 16: 1-6

23 Fischer D, Köllner V. Herzinsuffizienz, Kognition und Coping. PiD 2011; 12: 48-52

24 Härter M, Klesse C, Bermejo I et al. Unipolare Depression. Empfehlungen zur Diagnostik und Therapie aus der aktuellen S3- und Nationalen VersorgungsLeitlinie „Unipolare Depression“. Dtsch Arztebl Int 2010; 107: $700-708$

25 Whooley MA, Avins AL, Miranda J, Browner WS. Case-finding instruments for depression. J Gen Intern Med 1997; 12: 439-445

26 Herrmann-Lingen C, Buss U, Snaith R. Hospital Anxiety and Depression Scale - Deutsche Version (HADS-D). Manual. 3. Aufl. Bern: Hans Huber; 2011

27 Maercker A, Schützwohl M. Erfassung von psychischen Belastungsfolgen: Die Impact of Event Skala - revidierte Version (IES-R). Diagnostica 1998; 44: 130-141

28 Kalbe E, Kessler J, Calabrese P et al. DemTect: a new, sensitive cognitive screening test to support the diagnosis of mild cognitive impairment and early dementia. Int J Geriatr Psych 2004; 19: 136-143

29 Maercker A. Systematik und Wirksamkeit der Therapiemethoden. Posttraumatische Belastungsstörungen 2009; 3: 138-146

30 Margraf J. Kosten und Nutzen der Psychotherapie. Heidelberg: Springer Verlag; 2009

31 Markland D, Ryan RM, Tobin VJ et al. Motivational interviewing and self-determination theory. J Soc Clin Psychol 2005; 24: 811-831

32 Sniehotta FF, Schwarzer R, Scholz U et al. Action planning and coping planning for long-term lifestyle change: theory and assessment. Eur J Soc Psychol 2005; 35: $565-576$

33 Reese C, Mittag O, Bengel J et al. Praxisempfehlungen für psychologische Interventionen in der Rehabilitation: Koronare Herzerkrankung. Im Internet: http://www. uniklinik-freiburg.de/aqms/live/Praxisempfehlungen. html; Stand: 05.02.2014

34 Kurowski V, Kaiser A, von Hof K et al. Apical and midventricular transient left ventricular dysfunction syndrome (tako-tsubo cardiomyopathy): frequency, mechanisms, and prognosis. CHEST 2007; 132: 809-816

35 Kono T, Sabbah HN. Takotsubo cardiomyopathy. Heart Fail Rev 2013, DOI: 10.1007/s10741-013-9404-9: 1-9

36 Wittstein IS, Thiemann DR, Lima JAC et al. Neurohumoral features of myocardial stunning due to sudden emotional stress. N Engl J Med 2005; 352: 539-548 


\section{CME-Fragen}

CME-Teilnahme

- Viel Erfolg bei Ihrer CME-Teilnahme unter http://cme.thieme.de

- Diese Fortbildungseinheit ist 12 Monate online für eine CME-Teilnahme verfügbar.

- Sollten Sie Fragen zur Online-Teilnahme haben, unter http:// cme.thieme.de/hilfe finden Sie eine ausführliche Anleitung.

1. Auf das Vorliegen welcher psychischen Störung könnten Symptome wie Palpitationen, Brustschmerzen und Atemprobleme bei Patienten, die in die Notaufnahme kommen, hinweisen?

a Depression

b. Panikstörung

c. Trichotillomanie

d. Anorexia Nervosa

e. Schizophrenie

2. Was trägt zur Aufrechterhaltung des Circulus vitiosus der Angst bei der Panikstörung bei?

a. zu viel körperliche Aktivität

b. externale Stressoren

c. Missinterpretation körperlicher Sensationen als gefährlich

d. lebensbedrohliche Konsequenzen einer Panikattacke

e. Atemkontrollübungen

3. Welche Medikamente sollten in der Behandlung psychischer Komorbiditäten bei Herzkrankheiten nicht verschrieben werden?

a. Trizyklische Antidepressiva

b. Benzodiazepine

c. Selektive Serotonin-Wiederaufnahmehemmer

d. L-Dopa

e. Hypericum

4. Welche Aussage in Bezug auf psychische Belastungen bei kardialen Erkrankungen ist falsch?

a. Sie sind eigenständige Risikofaktoren für die Entwicklung einer Herzerkrankung.

b. Sie begünstigen die „traditionellen“ Risikofaktoren.

c. Sie wirken sich negativ auf die Lebensqualität und den Krankheitsverlauf aus.

d. Sie gefährden die Adhärenz

e. Sie senken das Risiko einer Rehospitalisierung.

5. Wie hoch ist die Prävalenz von klinischer Depression bei Patienten mit KHK?

a. $5-10 \%$

b. $15-20 \%$

c. $20-25 \%$

d. $25-30 \%$

e. $35-40 \%$

6. Wie kann man den Zusammenhang von Herz und Psyche beschreiben?

a. Wenn Bekannte an einer psychischen Störung leiden, hat man ein höheres Risiko, dass sich eine Herzinsuffizienz entwickelt.

b. Psychische Beschwerden überdecken eventuelle kardiale Erkrankungen.

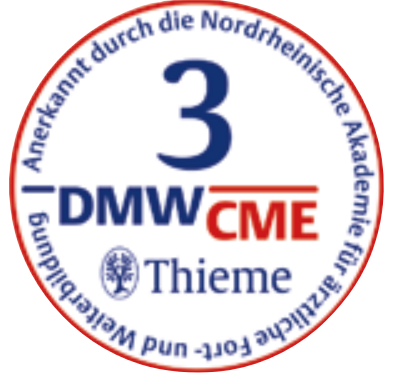

c. Sobald die Symptome der organischen Erkrankung behandelt sind, verschwinden die psychischen Belastungen automatisch.

d. Der Zusammenhang ist bidirektional: Psychische Belastungen wirken sich auf die Entstehung und den Verlauf von Herzkrankheiten aus und umgekehrt.

e. Psychische Beschwerden können den Verlauf von Herzerkrankungen nicht beeinflussen.

7. Was zählt nicht zu den Gefahren psychischer Störungen für das Krankheitsmanagement?

a. verspätete Inanspruchnahme ärztlicher Hilfe

b. verminderte Therapieadhärenz

c. kostspielige Überdiagnostik

d. ein erhöhtes Risiko für kardiale Ereignisse

e. verminderte sexuelle Aktivität

8. Wie sollte man mit Personen verfahren, deren Angehörige von einer kardiologischen Erkrankung und einer psychischen Störung betroffen sind?

a. Man sollte sie hinsichtlich kognitiver Defizite untersuchen.

b. Man sollte den Kontakt zu ihrem Angehörigen begrenzen, damit sie keinen schlechten Einfluss auf den Patienten ausüben.

c. Man sollte sie in das Beratungs-und Therapieangebot integrieren.

d. Man sollte ihnen Screening-Fragebögen mitgeben, um sich selbst auf mögliche Störungen zu testen.

e. Man sollte ihnen raten, eigene herzbezogene Symptome stärker zu beachten.

9. Das Risiko einer Depression beträgt bei Frauen unter 60 Jahren nach akutem Myokardinfarkt bis zu

a. $10 \%$

b. $20 \%$

c. $30 \%$

d. $40 \%$

e. $50 \%$

10. Bei welcher Patientengruppe mit Verdacht auf akutes Koronarsyndrom sollte die Diagnose Takotsubo/Stresskardiomyopathie in Erwägung gezogen werden?

a. bei Männern mit auffälligem Koronarangiografiebefund

b. bei menopausalen Frauen mit familiärer und beruflicher Doppelbelastung und auffälligem Koronarangiografiebefund

c. bei postmenopausalen Frauen mit kürzlich aufgetretenem belastenden Lebensereignis ohne Auffälligkeiten in der Koronarangiografie

d. bei postmenopausalen Frauen mit bevorstehendem belastenden Lebensereignis und Auffälligkeiten in der Koronarangiografie

e. bei Frauen mit postpartaler Depression und auffälligem Koronarangiografiebefund 


\section{Angaben zur Person}

Name, Vorname, Titel:

Straße, Hausnr.:

PLZ, Ort:

Anschrift: $\square$ privat $\square$ dienstlich

Ich bin Mitglied der Ärztekammer (bitte Namen der Kammer eintragen):

Jahr meiner Approbation:

Ich befinde mich in der Weiterbildung zum:

Ich habe eine abgeschlossene Weiterbildung in ...

(bitte Fach eintragen):

Ich bin tätig als: $\square$ Assistenzarzt $\square$ Oberarzt $\square$ Chefarzt $\square$ niedergelassener Arzt $\square$ Sonstiges

Ich bin DMW-Abonnent: $\square$ ja $\square$ nein

Falls nein: ich habe den Fragebogen aus / von:

$\square$ Thieme-connect $\square$ Kollegen $\square$ der Klinik $\square$ einer Bibliothek

$\square$ Sonstiges

Lernerfolgskontrolle (Eine Frage pro Antwort ankreuzen)
1. $\square$ A $\square$ B $\square$ C $\square$ D $\quad \square$ E
2. $\square \mathrm{A} \quad \square \mathrm{B} \quad \square \mathrm{C} \quad \square \mathrm{D} \quad \square \mathrm{E}$
3. $\square$ A $\square$ B $\square$ C $\square$ D $\quad \square \mathrm{E}$
4. $\square$ A $\square$ B $\square$ C $\square$ D $\square$ E
5. $\square \mathrm{A} \quad \square \mathrm{B} \quad \square \mathrm{C} \quad \square \mathrm{D} \quad \square \mathrm{E}$
6. $\square$ A $\square$ B $\square$ C $\square$ D $\square$ E
7. $\square$ A $\square$ B $\square \mathrm{C} \quad \square$ D $\square$ E
8. $\square$ A $\square$ B $\square$ C $\square$ D $\square$ E
9. $\square \mathrm{A} \quad \square \mathrm{B} \quad \square \mathrm{C} \quad \square \mathrm{D} \quad \square \mathrm{E}$
10. $\square \mathrm{A} \quad \square \mathrm{B} \quad \square \mathrm{C} \quad \square \mathrm{D} \quad \square \mathrm{E}$

Ich versichere, dass ich die Beantwortung der Fragen selbst und ohne fremde Hilfe durchgeführt habe

Ort, Datum:

Unterschrift:

Bitte in dieses Feld Ihre DMW Abonnement-Nummer eintragen:

\section{Fragen zur Zertifizierung}

1. Das Thema des Beitrages kommt in meiner ärztlichen Tätigkeit $\square$ häufig vor $\square$ selten vor $\square$ regelmäßig vor $\square$ gar nicht vor

2. Bei diesem Thema habe ich $\square$ eine feste Gesamtstrategie $\square$ keine Strategie $\square$ noch offene Einzelprobleme

3. In Bezug auf das Thema des Beitrages

$\square$ fühle ich mich nach dem Studium des Beitrags in meiner Strategie bestätigt

$\square$ habe ich meine Strategie verändert:

$\square$ habe ich erstmals eine einheitliche Strategie erarbeitet

$\square$ habe ich keine einheitliche Strategie ableiten können

4. Wurden aus der Sicht Ihrer täglichen Praxis heraus wichtige Aspekte des Themas

nicht erwähnt: $\square$ ja, welche $\square$ nein

zu knapp abgehandelt? $\square$ ja, welche $\square$ nein

überbewertet? $\square$ ja, welche $\square$ nein

5. Verständlichkeit des Beitrages

$\square$ Der Beitrag ist nur für Spezialisten verständlich

$\square$ Der Beitrag ist auch für Nicht-Spezialisten verständlich

6. Beantwortung der Fragen

$\square$ Die Fragen lassen sich aus dem Studium des Beitrags allein beantworten

$\square$ Die Fragen lassen sich nur unter Zuhilfenahme zusätzlicher Literatur beantworten

7. Die Aussagen des Beitrages benötigen eine ausführlichere Darstellung

$\square$ zusätzlicher Daten

$\square$ von Befunden bildgebender Verfahren

$\square$ die Darstellung ist ausreichend

8. Wieviel Zeit haben Sie für das Lesen des Beitrages und der Bearbeitung des Quiz benötigt?

Zertifizierungsfeld (wird durch die DMW ausgefüllt)

Ihr Ergebnis:

Sie haben

von 10 Fragen richtig beantwortet.

Sie haben $\square$ bestanden und 3 Punkte erworben

$\square$ nicht bestanden $\square$ ungültig, weil:

Stuttgart, den Stempel/Unterschrift 